PACSnumbers: 52.77.Bn, 81.16.-c, 81.20.Hy, 81.20.Wk, 81.40.Np, 81.40.Pq, 81.65.Cf

\title{
Some Considerations of Creating Nanotechnology for Part Forming
}

\author{
O. I. Soshko, V. O. Soshko, and I. P. Siminchenko
}

Kherson National Technical University,

Department of Automation, Robotics and Mechatronics,

24, Berislavske Shose,

UA-73008 Kherson, Ukraine

This article discusses some aspects and problems of mechanoplasma treatment technology based on the mechanoplasma effect that occurs in the processes of intensification of metal machining (in particular, cutting including turning, friction and destruction) in lubricating-cooling technological means.

У цій статті розглядаються деякі аспекти та проблеми технології механоплазмового оброблення на основі механоплазмового ефекту, що виникає в процесах інтенсифікації механічного обробляння металів (зокрема, різання, включаючи обточування, тертя та руйнування) в мастильно-охолоджувальних технологічних засобах.

Key words: mechanoplasma effect, metal machining, cutting, turning, friction, destruction.

Ключові слова: механоплазмовий ефект, оброблення металу, різання, обточка, тертя, руйнування.

(Received 11 February, 2020; in revised form, 20 February, 2020)

\section{INTRODUCTION}

Despite the significant development of methods of forming without removing chippings, metal cutting remains the leading, final process providing the specified requirements for accuracy, quality of the processed surface and other operational characteristics.

Metal cutting is a complex process, including turning, friction and destruction. The continuous watering of a liquid with several 
tens of components of lubricant-cooling agents (LCA) dissolved and emulsified in it influences all these processes.

Undoubtedly, the further development of metal processing will largely depend on the effectiveness of the influence of LCA on metal workability, a complex indicator that determines the rate of tool run out, the magnitude of the cutting force and power consumption, tool temperature and surface quality. Meanwhile, there is a rather extensive material showing that despite the use of new continuously updated compositions of LCA, the complexity of machining continues to be large, and in many cases technological means do not yet provide the specified requirements for accuracy and surface quality as well as other operational characteristics of manufactured critical parts.

This state of the problem remains for decades, that is largely due to the ambiguity of ideas about the physical nature of the processes of contact interactions of the medium with a new electrically active metal structure formed due to physical impact, and this does not contribute to the efficiency of the search of new approaches to solve the problem under consideration.

Analysis of reviews, reports and articles makes it possible to highlight some areas of research; their implementation will overcome this difficulty and advance in solving this problem in the right direction [1].

\section{EXPERIMENTAL/THEORETICAL METHODS}

Among them, the most important, specific and interesting area of research is the possible initiation of activation processes in the chip formation zone of the initial electro passive surfactant medium (LCA) and its subsequent transformation by wave propagation of chemical reactions up to the formation of an electrically active ionized gas [2]. It was supposed that such a gas, consisting of hydrogen in its active forms, electrons, and neutral molecules, is ideally suited to perform the functions of a technological medium capable to improve optimally the workability of a metal [3]. Such a process becomes possible because the complex of physicochemical processes and phenomena accompanying the mechanical process creates the conditions necessary for the occurrence of chemical transformations of the initial hydrocarbon medium into radically active hydrogen plasma $[4,5]$.

Solving this complex problem requires setting new tasks: studying the phenomena of quantum diffusion of hydrogen particles in a narrow gap space of a crack with a pronounced polar effect at its apex, transportation of a medium in an inhomogeneous region of plastically deformable material in front of a crack apex, and reveal- 


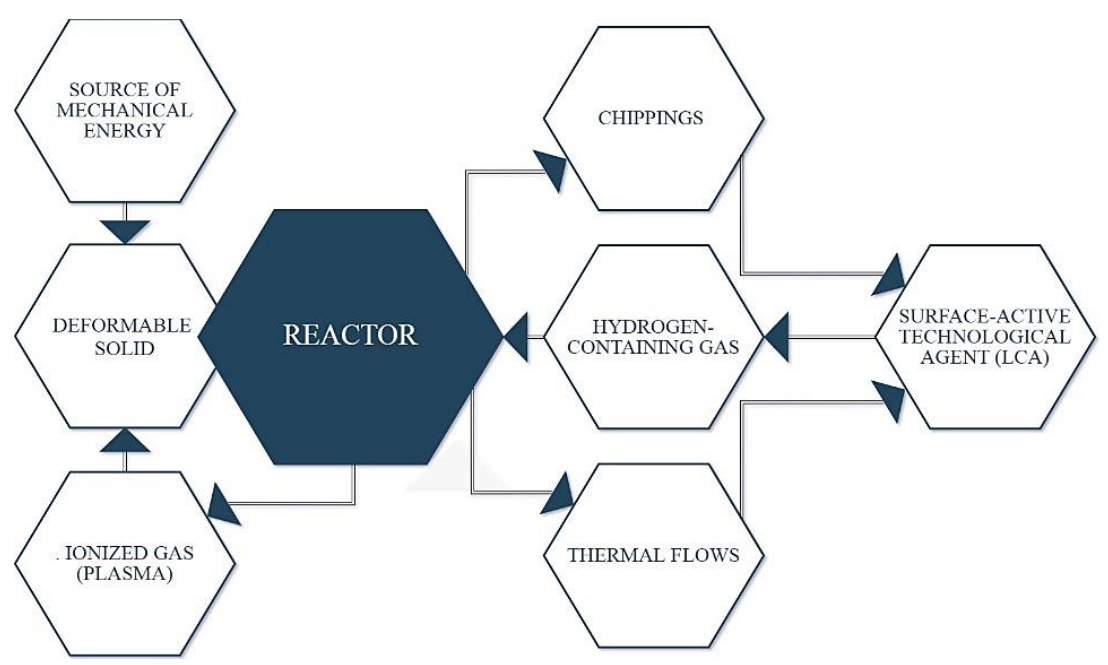

Fig. The scheme of positive feedback between quantum effects and chemical processes generated by a growing crack.

ing the nature of contact interactions of particles with electric charge and their influence on the processes of deformation and fracture, etc.

To solve these problems, contributing to a radical increase in the efficiency of the processing medium, it is necessary not only to transfer the interacting metal-medium system from the level of weak adsorption interaction of the body with the medium to a much stronger level-electrical interaction, but also to ensure timely flow of the medium into the adhesion overcome zone between atoms of a deformable material.

The practical aspect of this assumption was to use the properties of the resulting objects and materials of nanodimensional scale, which differ in extremely high aggressiveness from the properties of free atoms or molecules, as well as from the bulk properties of a substance consisting of these atoms or molecules, as a processing medium. In addition, as such a product has an electric charge, its interaction with the electromagnetic field that occurs in the working system 'machine-tool-part' allows for timely delivery of the activated substance to the plastically deformable area of the material, that is, directly to the rearrangement zone and breaking of the bonds of atoms. It was also assumed that such a problem could be solved only if high molecular compounds are used as the main additives to LCA [6]. This conclusion was justified by the specific properties of polymers, which are manifested when bonds are broken in the main polymer chain under the influence of various physical, chemical, and mechanical factors. In particular, polymers under the 
influence of such factors at the last stage of chemical reactions produce a product of a qualitatively changed physicochemical property and high reactivity. It was believed that the conditions that form in the cutting zone and in the slit space of the crack due to the accompanying destruction of various physical and chemical processes and phenomena of quantum nature are sufficient for the manifestation of specific features of polymers [7]. Consequently, in the case of the implementation of the proposed medium activation scheme, the possibility of the formation of a hydrogen plasma at a crack dead end as an medium performing the function of a technological tool, i.e., LCA. In turn, this should lead to the development of a new technology for obtaining new technological tools that contribute to solving practical problems of metal machining.

Some physicochemical laws of high reactivity of polymer additive to LCA.

The reasons for the high reactivity of the polymer additive are most pronounced in comparison with their low molecular weight counterpart. In relation to the problem under consideration, the main differences are as follows.

If we compare the detachment energy of a hydrogen atom in a series of saturated hydrocarbons (in many cases they make LCA), then, in comparative units, it will be 11 units for heptane, 3 units for polyethylene, and 2 units for polyisobutylene [8]. This means that the decomposition of polymer macrochains occurs at a much lower temperature than a molecule of a similar chemical nature. In addition, the destruction of the polymer on the hot surfaces of the chippings and tool, that is, the decomposition of the polymer, unlike low molecular weight substances, will proceed according to the free-radical chain mechanism, which gives a high rate of an elementary act of chemical transformation and a high concentration of the product formed [1, 9].

As known, among the physical factors that can initiate chemical reactions in polymers, thermal exposure occupies an important place, as it is the cause of one of the most important characteristics of polymers-their thermal stability. For example, the decomposition of polyethylene (PE) begins at a temperature of $290^{\circ} \mathrm{C}$, and at a temperature of $315^{\circ} \mathrm{C}$, the rapid formation of volatile products occurs [10]. Therefore, if polyethylene is used as an additive to LCA, then, the temperature at which its chemical transformations begin will be significantly lower than the temperature of heat fluxes in the cutting zone and the temperature of the chippings.

It should be noted one more important feature of the chemical transformations of the polymer, as additive to LCA. Unlike low molecular weight analogues, high molecular weight compounds even as a result of the first act of chain breaking give a high concentration 
of reactive products that cause further chemical reactions. In this case, the radicals move from one chain (or chain fragment) to another by detaching the hydrogen atom until they get close enough for them to recombine. Moreover, each radical is able to initiate a new radical reaction, leading to the breaking of about 10000 polymer chains [11]. The formation and accumulation of active products and relatively stable intermediates is an additional source of increasing the concentration of new active particles, because of which the reaction becomes self-accelerating, and the formation of a large concentration of ionized hydrogen. The peculiarity of the decay of the polymer macrochain lies in the fact that, as it is known from [8, 9], during the pyrolysis of the polymer and, in particular, $\mathrm{PE}$, in addition to the gas phase, a solid phase is also formed, namely, a polyconjugated polycyclic system consisting of graphitized aromatic rings [4]. In this regard, in the process of cutting, when using PE as an additive to LCA, carbon accumulation in the form of allotropic modification graphite is possible on the cutting surfaces of the tool [1]. Such a pyropolymer residue, firstly, can be a good lubricant that promotes the separation of friction surfaces, and secondly, a medium for the implementation of permanent carbonization of the tool cutting edges. Both the first feature of the LCA polymer additive and the second one are that both of them will contribute to a significant increase in the tool wear resistance, as well as a reduction in the work spent on friction of the front and back surfaces of the tool with the processed material and chippings.

Thus, the purpose of the polymer additive is to influence not only the main energy costs of the cutting process (plastic deformation and friction), but also contribute to the achievement of the main goal of machining, namely, to obtain parts with a given accuracy and surface quality while obtaining certain economic results.

The following should be noted. It is known that the reactivity of a functional group does not depend on whether it is attached to a polymer chain of any length or to be part of a small molecule of a low molecular-weight substance [8]. This principle is qualitatively observed, but the quantitative comparison shows significant differences in the reactivity of low and high molecular weight compounds of similar chemical nature. Therefore, when considering the chemical reaction process, two questions arise in order to obtain the desired substance. The first one is as follows: 'Is a reaction possible under the given conditions?' Equally important is the second question: 'Under what conditions will the reaction precede fast enough so that this process has practical significance?' Returning to our case, it can be argued that in the conditions of the cutting zone, both low- and high-molecular compounds that make up the LCA will decompose. For this, the formed conditions in the process of sepa- 
rating the chippings from the work piece are sufficient for a positive answer to the first and second question [1]. At the same time, because of chemical transformations of a low molecular weight substance, the rate of formation of the desired substance and its concentration are so insignificant that they do not have a significant effect on the processes of metal deformation and fracture during cutting, and, consequently, such a chemical process has no practical meaning.

Crack as a factor forming conditions for the chemical activation of the medium and its transportation to the fracture zone.

Since the breaking of the interatomic bonds of the body is localized in places of stress concentration of different origin, but mainly in the microregions of the cracks adjacent to the tops, in the article it is considered the situation that develops in such areas due to the possible influence of the medium. In this case, it is not a question of defects (microcracks) emerging on the surface of the deformable metal, but only of growing microcracks at the vertices of which new surfaces are opened, i.e., located and developing in close proximity to the cutting edge of the tool. We will consider such a microcrack having a vee shape with a collar descending to the interatomic distance as a source of subsequent destruction.

The results of studies of the kinetics of the cutting process, confirmed by experiment [12], show that, at the microscale, the chip does not contact the tip of the tool cutter, creating a reaction slot mating with the cavities of the crack [7]. Due to mechanical action on the material, a crack grows, its juvenile surfaces are exposed, and at the same time, various physical processes and phenomena are generated in the slot space.

Consequently, the zone limited by the cavity of the crack, the wedge of the tool and the tip of the crack can be represented as a kind of reactor. In its space under the influence of various processes and phenomena accompanying the destruction, the hydrogencontaining gas mixture formed at the first stage of transformations is transformed into radically active hydrogen plasma [1].

According to the proposed scheme, the hydrocarbon gas mixture resulting from the destruction of the polymer component of the LCA will continuously flow and accumulate in the space bounded by the crack tip, its 'banks' and the cutting edge of the tool. In this reaction zone the final formation of hydrogen plasma occurs. The ionizers in this process are juvenile catalytic active new crack surfaces, exoelectronic emission, as well as self-accelerating gasdischarge processes that occur in the crack cavity [2].

Based on this conceptual position, we will consider how surfaces are formed at the moment of destruction of a solid body, and what their physicochemical properties are. 
Today, there is no longer any doubt that the mechanical effect leading to the growth of a crack in a solid and the formation of new surfaces is accompanied by an outbreak of the chemical transformation of the medium [11]. In fact, in this case the growth of the crack plays the role of a trigger mechanism, which includes various chemical reactions. One of them will be initiated by new surfaces. Such a freshly formed surface is not ideal; point defects periodically are located on it, the most electrically active centres, such as the vertices, ribs and edges of crystals, steps of chips, pores, and then less active, in accordance with the type of symmetry and spatial arrangement of atoms on the surface. These surface elements can be represented as microelectrodes capable of taking part on the one hand, in various physical and chemical processes, and on the other, as acting accelerating or inhibiting potentials when electrons and ions contact and migrate to the crystal surface. Therefore, a mosaic, catalytically active surface is capable of activating the chemical reactions occurring on it, sometimes even with zero activation energy. So, for example, the fact of hydrogen catalysis on a clean surface of iron was established even at a temperature of $253^{\circ} \mathrm{C}$ [13]. By analogy with this, it can be assumed that during contact interactions of the constituents of the gas mixture, chemicals with the surfaces of the crack, a chain of chemical transformations of substances will also occur with the formation of hydrogen-containing plasma at the final stage, i.e., ionized gas.

The ability of catalytically active metal surfaces to generate active forms of hydrogen in volume was established in the early works of Langmuir. Obviously, in this case, the surface heterogeneity primarily causes interaction with the first layer of elements of the gas mixture that comes into contact with it. However, since a large number of lattice defects (vacancies, dislocations, grain boundaries, subgrains, and phases) with sizes from several angstroms to $100 \AA$ come to the surface, adsorption of the following gas layers will occur. In addition, such defects can apparently be modelled in the form of channels, which can serve as pathways for the predominant diffusion of hydrogen.

The reaction zone has another extremely important property. The exclusivity of this property is that on the contact surfaces of the tool with the processed material in the chip formation zone, some electrons in the process of plastic deformation and friction acquire kinetic energy sufficient to overcome the potential barrier of their separation from the metal boundary, which leads to thermal current [14]. Since the main current carriers, electrons, move from the heated region (the tool blade) to the cold (processed metal), the work piece will be enriched with charge carriers, electrons, and acquire a negative space charge, and the instrument, depleted in elec- 
trons, will be charged positively. This process proceeds continuously, as well as the 'pumping' of electrons in one direction from the tool to the material being processed, and an electric current will flow in the 'machine-tool-work piece' system. In this regard, in the reaction zone, there will be a separation of the direction of flow of positively and negatively charged hydrogen particles: the tool will attract particles of negative electric charge, and protons will begin to move in the direction of the surface of the crack tip. After passing through the surface of positively charged hydrogen particles, they will be carried away by the conduction electrons of the treated metal, moving in an electric field at high speed. The drag of protons by conduction electrons is so great that positively charged particles begin to move, as a rule, towards the anode, against the field, like particles with a large negative charge [15, 16]. This process, called the electronic 'wind' effect, can provide the speed of proton movement along the shear layer at a speed exceeding the crack growth rate [3].

Finally, exoelectronic emission plays a significant role in the formation of hydrogen plasma in the reaction region. This phenomenon occurs because electrons escape from the collar of the crack during its growth with high intensity (6.10 pulses per minute) and energy $\left(10^{2} \mathrm{eV}\right)$ [17], exceeding the ionization energy of the hydrogen atom $(13.595 \mathrm{eV})$. This means that conditions are created in the reaction zone for impact ionization of hydrogen molecules and residual gas hydrocarbons. Moreover, the processes proceeding from the appearance of primary ions to the formation of new as well as molecular products and free radicals complete in just $10^{-5}$ seconds [15].

The notions of the physicochemical nature of the activation of the initial medium are confirmed not only by modern theoretical studies, but also by the practical result of their use. Therefore, for example, if a metal plate is introduced into the ionized hydrogen stream, its surface will quickly melt and a weld pool will form. In this case, the flame temperature is about $3700^{\circ} \mathrm{C}$. On this principle, an industrial welding method has also been developed, which is called 'atomic arc welding'. In addition, the technology of plasma cutting of metal is known, when a narrow stream of ionized hydrogen is directed to the metal, melting it in a thin layer. Thus, because of impact ionization, chemical reactions on the catalytically active surfaces of the crack, and self-accelerating reactions in the reaction zone, a high concentration of a water conduit in the ionized state will begin to form at a high speed. Subsequently, under the action of the electric field of the system, as well as the negative charge concentrated at the crack tip [1], the flow of protons carrying a positive electric charge will rush through the narrow gap to 
the crack tip. When approaching its crack tip, the proton flux is divided into two parts. One part of the protons collides with the flow of exoelectronic emission and, because of interaction with electrons, recombines with the release of thermal energy (from 60 to $120 \mathrm{kcal} / \mathrm{mole}$ ). The second part of the protons, passing through the surface of the collar of the crack, freely trans- and intergranular moves along the shear layer due to the effect of electronic 'wind'. In this case, some diffusing protons become trapped by hydrogen traps, in which they also recombine with the release of thermal energy.

The provisions expressed here were fully confirmed by the results of experimental and theoretical studies, as well as by practice data, this is reflected in some publications [1-5, 7].

\section{RESULTS AND THEIR DISCUSSION}

It is important to note that a significant feature of the proposed model is the real possibility of a positive feedback between the growing crack and the medium: the developing crack creates conditions in the slot space for the medium to be activated before the hydrogen plasma, and the resulting plasma has a positive effect on its development (Fig.). In this case, the final activation processes take place in a narrow slit space with a pronounced polar effect at the crack tip with the formation of chemical transformations among pre-hydrogen plasma at the last stage.

Thus, for the implementation of mechanoplasma treatment, the process of activation of a polymer-containing surface-active medium and its transformation into radically active hydrogen plasma should include the following stages.

1. Adsorption of polymer additives to LCA, consisting of hydrocarbon molecules and its chemical compounds, on the hot surfaces of the cutting zone.

2. Thermal degradation of macromolecular compounds.

3 . The chain reaction of the macrochain, initiated by the active products formed during its pyrolysis.

4. Desorption of reaction products-a gas mixture in various active forms of hydrogen, as well as a homologous series of aliphatic saturated hydrocarbons.

5. Diffusion of the resulting activated products into the reaction gap.

6. Heterogeneous and homogeneous catalytic reactions on juvenile freshly formed surfaces and in the space of the reaction gap with the formation of ionized hydrogen-containing gas.

7. Recombination of protons in the space between the cracks.

8. Impact ionization of a hydrogen-containing gas by an electron 
avalanche with the formation of a hydrogen plasma.

\section{CONCLUSION}

The problem of the need for the development of metalworking and the most important research tasks put forward in this connection, which are based on the fundamental laws of physics of the cutting process and constantly emerging and developing new frontier areas of science, are formulated. The main objective of the research is related to the knowledge of the laws of such extremely complex in terms of diversity and extremeness of the processes conditions, which is the process of metal cutting during continuous watering of a liquid with several tens of components dissolved and emulsified in it. The solution of this problem should lead to an understanding of the physical nature of the phenomena that accompany this process, and based on this, it is possible to develop new approaches to create the optimal technology for the shaping of parts. One of them may be the proposed technology of mechanoplasma processing, which at the same time combines not only the possibility of intensifying the process of parts manufacturing at its optimum cost-effectiveness, but also obtaining products with operational properties, which are adjustable in a certain range.

A model of a radical improvement in metal workability and solution methods are offered. Its implementation is possible if a plasma effect is formed in the reaction zone between the tool wedge and the crack tip. Positively charged hydrogen particles diffusing from the plasma and accelerated by the electric field interact with the real, electrically active structure of the material and, as a result, a large thermal energy is released.

This phenomenon leads to a significant relief of the deformation and fracture processes. The manifestation of the plasma effect depends on the presence in the medium of hydrogen compounds, the atoms of which are part of any technological tool. However, the greatest effect is achieved with a maximum degree of ionization and hydrogen concentration in the crack cavity. Conditions for formation in the reaction zone are created only if a high molecular weight compound is present in the composition of the technological means. This conclusion is of great practical importance, since direct recommendations from here should be sought for effective additives to LCA among polymer compounds that give reactive forms of hydrogen and carbon in the chain of chemical transformations of their macrochains.

The experimental results and data from industrial enterprises convincingly demonstrate the undoubted advantage of mechanoplasma processing. The formation of parts in this case is character- 
ized by high machinability, which is characterized by a decrease in tool wear and cutting force, an increase in tool life and quality of the machined surface, as well as the fact that the process proceeds at high dimensional accuracy with the achievement of a large economic effect.

To implement the technological process of mechanoplasma treatment, it is necessary to use the unique properties of objects and materials of the nanometre range, which are highly active than the properties of free atoms or molecules, as well as from the bulk properties of compounds and substances consisting of the same atoms or molecules.

It should be noted that the plasma effect is no less pronounced during technological processes of processing other solids. For example, a significant effect is observed when grinding solid bodies of inorganic nature, and drilling solid bodies of geological rocks with a high degree of strength, processing silicate glasses and semiconductor crystals, etc.

The authors sought to show in the article the great promise of using mechanoplasma treatment of solids, and also bearing in mind the importance and complexity of the problem, they considered it possible to express some hypothetical thoughts.

Detailed information that can be used to develop a highly efficient technology for mechanoplasma processing is given in the brochure [18].

\section{REFERENCES}

1. O. I. Soshko and V. O. Soshko, Progress in Physics of Metals, 20, No. 1: 96 (2019); https://doi.org/10.15407/ufm.20.01.096

2. O. I. Soshko and V. O. Soshko, Metallofizika i Noveishie Tekhnologii, 39, No. 1: 117 (2017) (in Russian); doi:10.15407/mfint.39.01.0117

3. A. I. Soshko and V. A. Soshko, Mechanochemical Processing of Metals (Saarbruchen: LAMBERT Academic Publishing: 2015).

4. A. I. Soshko and V. A. Soshko, Lubricating and Cooling Technological Means in Metal Machining (Kherson: Oldie-plus: 2008), part 1 (in Russian).

5. A. I. Soshko and V. A. Soshko, Lubricating and Cooling Technological Means in Metal Machining (Kherson: Oldie-plus: 2008), part 2 (in Russian).

6. A. I. Soshko, Mechanical Processing of Metals: Polimery v Tekhnolog icheskikh Protsessakh Obrabotki Metallov (Kiev: Naukova Dumka: 1977), p. 16 (in Russian).

7. A. I. Soshko, V. A. Soshko, and I. P. Siminchenko, Nanosistemi, Nanomateriali, Nanotehnologii, 16, Iss. 3: 451 (2018); https://doi.org/10.15407/nnn.16.03.425

8. V. N. Kuleznev and V. A. Shershnev, Chemistry and Physics of Polymers (Moscow: Vysshaya Shkola: 1988) (in Russian).

9. I. I. Tugov and G. I. Kostrykina, Chemistry and Physics of Polymers (Mos- 
cow: Chemistry: 1989) (in Russian).

10. N. Grassie and J. R. MacCallum, Journal of Polymer Science. Part A: General Papers, 2, Iss.2: 983 (1964).

11. A. M. Zanin, D. P. Kiryukhin, I. M. Barkalov, and V. I. Goldanskiy, Pisma $v$ ZhETF, 33, Iss. 6: 336 (1981) (in Russian).

12. E. J. A. Armarego and R. H. Brown, Obrabotka Metallov Rezaniem [Processing of Metals by Cutting] (Moscow: Mashinostroenie: 1977) (Russian translation).

13. S. Z. Roginsky, Theoretical Foundations of Heterogeneous Contact Catalysis (Moscow: AN SSSR: 1936), vol. 1, 2 (in Russian).

14. V. N. Poduraev, Cutting of Hard Materials (Moscow: Vysshaya Shkola: 1974) (in Russian).

15. R. W. Christy and A. Pytte, Stroyenie Veshchestva: Vvedenie v Sovremennuyu Fiziku [The Structure of Matter: Introduction to Modern Physics] (Moscow: Nauka: 1969) (Russian translation).

16. T. N. Trofimova, Course of Physics (Moscow: Vysshaya Shkola: 2000) (in Russian).

17. A. S. Akhmatov, Molecular Physics of Boundary Friction (Moscow: GIFML: 1963) (in Russian).

18. A. Soshko et al., Polymer-Containing Cutting Fluids (Lviv: Kamenyar: 1986) (in Russian). 